\title{
Diagnosis of Bilateral Tonsil Cancers via Staging PET/CT: Case Report and Review
}

\author{
Edward M. Mannina, ${ }^{1}$ Sunanda M. Pejavar, ${ }^{1}$ Christine M. Glastonbury, ${ }^{2}$ \\ Annemieke van Zante, ${ }^{3}$ Steven J. Wang, ${ }^{4}$ and Sue S. Yom ${ }^{1}$ \\ ${ }^{1}$ Department of Radiation Oncology, University of California San Francisco, 1600 Divisadero Street, P.O. Box 1708, Suite H-1031, \\ San Francisco, CA 94143, USA \\ ${ }^{2}$ Department of Radiology \& Biomedical Imaging, University of California San Francisco, 505 Parnassus Avenue, P.O. Box 0628, \\ Room L-358, San Francisco, CA 94143, USA \\ ${ }^{3}$ Department of Anatomic Pathology, University of California San Francisco, 1600 Divisadero Street, P.O. Box 1785, Room B231, \\ San Francisco, CA 94143, USA \\ ${ }^{4}$ Department of Otolaryngology-Head and Neck Surgery, University of California San Francisco, 2233 Post Street, 3rd Floor, \\ P.O. Box 1225, San Francisco, CA 94115, USA
}

Correspondence should be addressed to Sue S. Yom, yoms@radonc.ucsf.edu

Received 9 January 2011; Accepted 26 May 2011

Academic Editor: Shakeel Riaz Saeed

Copyright () 2011 Edward M. Mannina et al. This is an open access article distributed under the Creative Commons Attribution License, which permits unrestricted use, distribution, and reproduction in any medium, provided the original work is properly cited.

\begin{abstract}
Diagnostic workup of metastatic head and neck squamous cell carcinoma of unknown primary site has traditionally included $\mathrm{CT}$ and/or MRI imaging and endoscopic biopsies. Routine bilateral tonsillectomy is highly controversial and the role of PET/CT is evolving, both for identification of potential primary sites and the detection of distant metastases. We report a case of cervical nodal metastasis of squamous cell carcinoma from an unknown primary site, in which dual-modality PET/CT led to the unexpected diagnosis of synchronous bilateral tonsillar cancers. In addition, PET/CT correctly distinguished pulmonary sarcoidosis from metastatic disease in this patient.
\end{abstract}

\section{Introduction}

The standard workup for a head and neck squamous cell carcinoma of unknown primary site (CUPS) includes physical exam, chest imaging, CT or MRI of the head and neck region, and panendoscopy with biopsies of potential primary sites. Given that a high proportion of occult tumors are located in the palatine tonsils, diagnostic unilateral tonsillectomy is frequently recommended. Bilateral tonsillectomy has been proposed but remains controversial. This case illustrates that skilled interpretation of PET/CT, incorporating a diagnosticquality anatomic imaging component, can correctly identify clinically inapparent synchronous tonsil cancers and assist in the evaluation for distant disease.

\section{Case Report}

A 57-year-old man presented with a flu-like syndrome and right neck swelling. Fine-needle aspiration of the right neck mass revealed squamous cell carcinoma, and a CT scan of the head and neck revealed an enlarged right jugulodigastric lymph node $(2.9 \times 2.5 \mathrm{~cm})$. A CT scan of the chest showed mediastinal and bilateral hilar lymphadenopathy with bilateral interstitial nodular opacities in the upper lobes. A diagnostic PET scan showed the right jugulodigastric node to have a standardized uptake value (SUV) of 5.98 with bilateral oropharyngeal radiotracer activity. Surprisingly, radiotracer uptake in the oropharynx was higher in the left tonsil compared to the right. The patient underwent two rounds of panendoscopy with biopsies which revealed, respectively, mild dysplasia of the right tonsil and a friable and nodular inferior border of the right tonsil containing carcinoma in situ. Diagnostic right tonsillectomy showed extensive squamous cell carcinoma in situ with a high suspicion of invasion. The left tonsil was specifically noted to be clinically unremarkable.

A repeat PET/CT was performed for the purposes of radiotherapy planning and this study confirmed the presence 


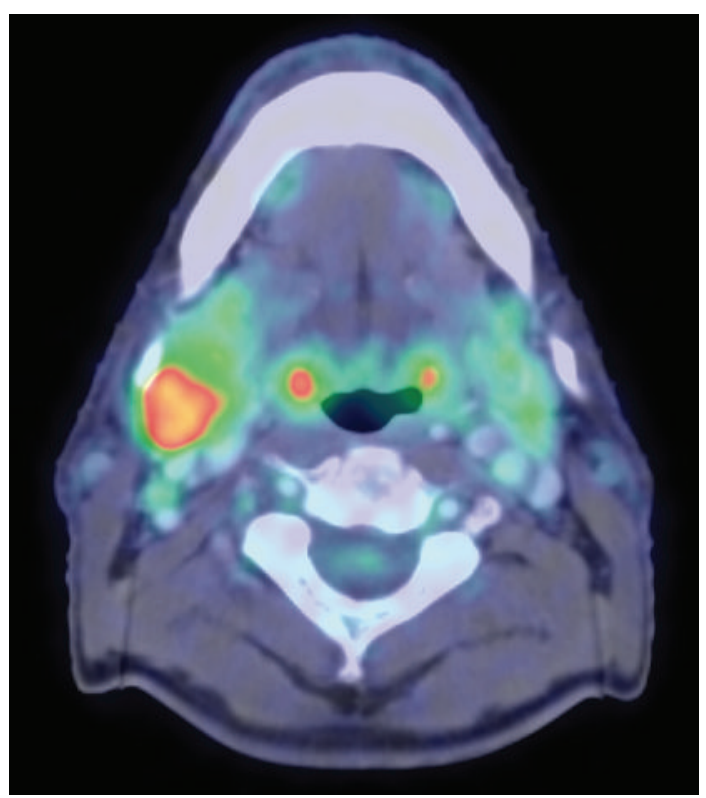

Figure 1: Axial view from fused PET/CT study demonstrates an enlarged right jugulodigastric node with heterogeneous but overall increased FDG avidity with peak SUV of 7.3.

of a $3 \mathrm{~cm}$ right neck level II lymph node with an SUV of 7.3, with central necrosis (Figure 1). A diagnostic head and neck contrast-enhanced CT was included as part of the $\mathrm{PET} / \mathrm{CT}$ study, as is our institutional practice and review of the CT elicited concern for extracapsular spread. FDGavidity was again noted bilaterally in the oropharynx, with the intact left tonsil showing a maximum SUV of 8.7 as compared to maximum SUV of 6.6 in the tonsillar bed on the right (Figure 2(a)). However, the left tonsil was noted to be unusually bulky and irregular on the CT portion of the imaging study (Figure 2(b)). The PET/CT also demonstrated mild, symmetric FDG avidity in the mediastinal and hilar regions, which was considered more consistent with an inflammatory rather than neoplastic process, given the low FDG uptake (Figure 3). Highresolution chest $\mathrm{CT}$ also suggested sarcoidosis. Due to the possibility that the asymmetric uptake and enlargement of the intact left tonsil might have been caused by the recent right tonsillectomy, the patient underwent a diagnostic left tonsillectomy. While the appearance of the left tonsil was still normal on examination, pathologic analysis revealed extensive in situ and invasive squamous cell carcinoma (Figure 4(a)). Immunohistochemical staining for p16 was diffusely and strongly positive (Figure 4(b)). Concurrent cisplatin was recommended due to the radiographically identified extracapsular extension, but the patient chose cetuximab in conjunction with intensity-modulated radiotherapy. Radiation treatment targeted the bilateral tonsillar beds and the right neck lymph node. The prescription dose was 6996 cGy, delivered over 33 fractions of treatment, with weekly localization using conebeam CT imaging to position the patient on the radiation therapy table. The concurrent cetuximab was delivered over 8 weekly infusion cycles and was well tolerated, with development of a moderate skin rash

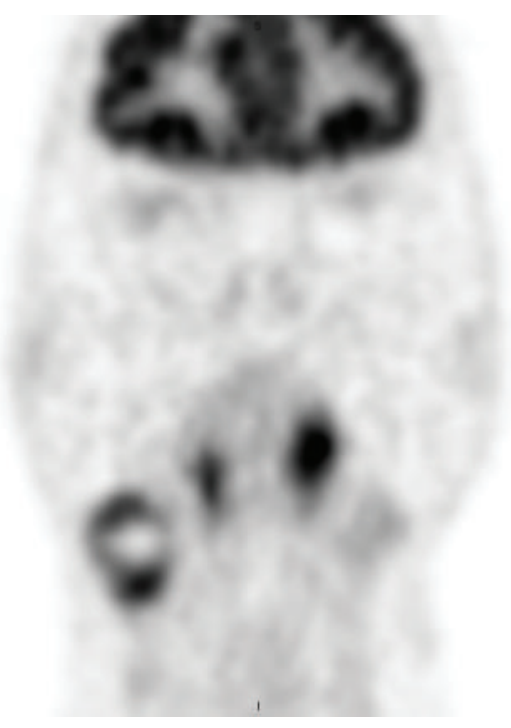

(a)

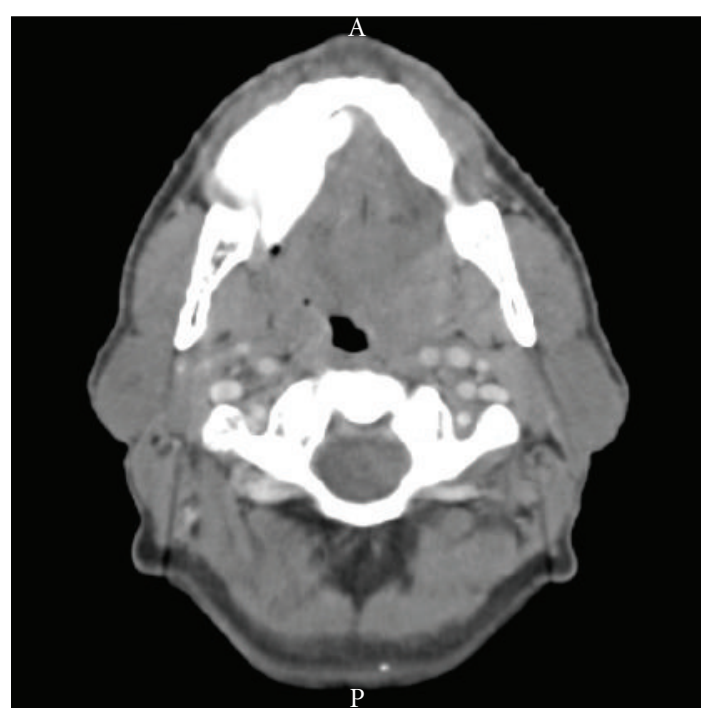

(b)

Figure 2: (a) Coronal FDG PET showing bilateral but asymmetric tonsillar FDG avidity. The patient had undergone diagnostic right tonsillectomy prior to this PET/CT study. However, peak SUV in the contralateral left palatine tonsil measured 8.7. (b) Axial CT showing pronounced left-sided tonsillar enlargement corresponding to the high FDG avidity.

as is typical for this class of targeted therapy. After 2 years of followup, the patient is well with no evidence of recurrent cancer and his sarcoidosis remains stable.

\section{Discussion}

The exact diagnostic workup for CUPS remains a matter of debate. Examination under anesthesia is mandatory, but biopsy procedures are variable. Because a significant percentage of CUPS arise from the palatine tonsil [1-4], a typical recommendation is that an ipsilateral tonsillectomy be included in the standard workup [2, 3, 5-7]. However, a 


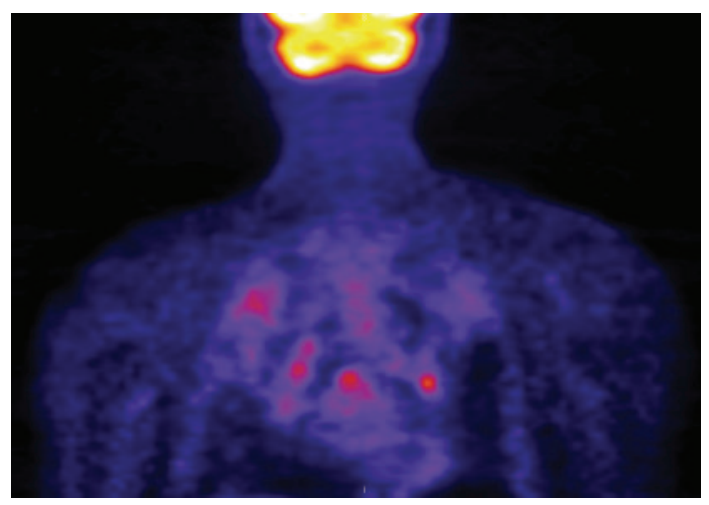

FIGURE 3: Coronal FDG-PET shows mild mediastinal and bilateral hilar FDG uptake, which is nonspecific but consistent with sarcoidosis.

case series published by Koch et al. [8] found that the incidence of bilateral tonsillar cancers approaches $10 \%$, leading to a recommendation for routine diagnostic bilateral tonsillectomy based on the goals of early control of all primary disease [5], avoidance of radiation to healthy tissues $[3,6]$, improved surveillance ability [6], and improved overall success rates $[9,10]$. Yet despite probable benefit in a select number of patients, the practice of bilateral tonsillectomy remains controversial.

The optimal imaging workup likewise remains undefined. A retrospective review found that PET/CT was superior to CT, MRI or whole body PET in primary site detection [9]. Compared to CT alone, PET/CT has higher sensitivity $[4,11]$, higher negative predictive value [4], and comparable [11] or inferior specificity [4] in the investigation of head and neck CUPS. Studies have estimated that the sensitivity of PET/CT may range from $66-87.5 \%$ with a specificity of $70-92.9 \%[4,11-13]$. A positive predictive value of $88.8 \%$ has been reported [12].

A retrospective review by Cianchetti et al. [7] included a proposed diagnostic algorithm for CUPS of the head and neck, including a complete physical exam, CT and/or MRI, panendoscopy with biopsies, PET/CT based on "indeterminate findings," and unilateral or bilateral tonsillectomy in patients with extant lymphoid tissue [7]. In contrast, some authors have advocated for upfront PET/CT in order to direct the choice of biopsy sites at the time of panendoscopy, thereby increasing accuracy in detecting singular or synchronous primary cancers $[14,15]$. We agree with the latter recommendation.

Of note, this patient was a lifelong nonsmoker and showed strong immunohistochemical staining for p16, consistent with association with high-risk human papillomavirus infection [16]. HPV-related oropharyngeal cancers treated with chemoradiation carry a superior prognosis, which is retained despite traditional indicators of aggressiveness such as regional nodal metastasis $[17,18]$. One report did not find an increased propensity for bilateral tonsillar cancer in specimens that were positive for p16 immunohistochemistry and HPV-16 in situ hybridization [19]. However, other reports have speculated on the possibility of HPV-related field

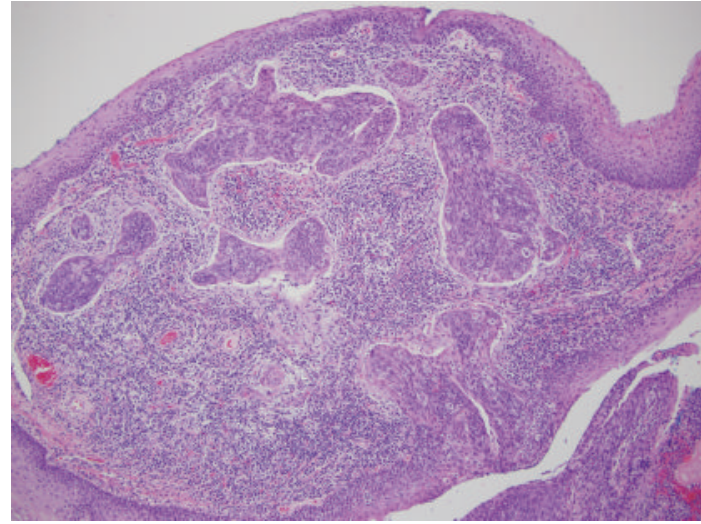

(a)

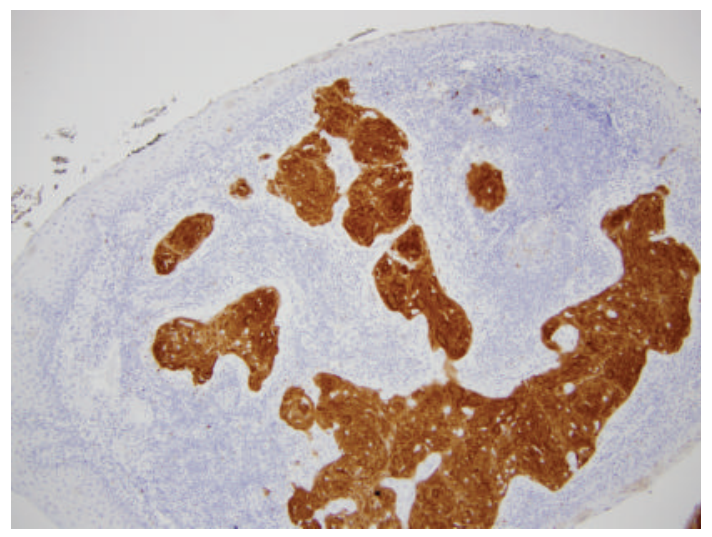

(b)

Figure 4: (a) Hematoxylin and eosin stained section of the left tonsil showing invasive squamous cell carcinoma, magnification 100X (b) Immunohistochemical stain for p16 performed on a serial section, magnification $100 \mathrm{X}$.

cancerization in Waldeyer's ring [20] or an HPV-related predisposition to the development of bilateral disease spread [21].

Finally, PET/CT findings may result in changes to staging or alteration of radiation therapy target volumes [13,22-24]. A large prospective, blinded study specifically noted changes to the gross tumor volume, the extent of regional disease, the prescribed dose of radiation and/or the selection of treatment modality, leading to nearly a third of the patients' management being significantly altered [22]. One study found higher control rates and favorable toxicity profiles when utilizing PET/CT to guide radiotherapy [25].

Oncologists should be aware of the limitations of PET/CT in cases of head and neck CUPS. A high rate of false positive findings with PET/CT in the postoperative and preradiotherapy period has been shown [26]. Conditions such as infection, healing, or localized inflammatory processes as well as foreign bodies (catheters, prostheses) have been shown to produce false positive results on PET/CT [27]. Though rare, the so-called "sarcoid-like reaction to malignancy" should be considered when interpreting PET/CT [28]. F-18 fluorothymidine may have an advantage over 
F-18 fluorodeoxyglucose PET/CT scans for distinguishing sarcoidosis from malignancy [29].

Most importantly, the value of obtaining imaging studies of appropriate quality and technique should not be understated. While PET alone may be adequate for CUPS workup [30], other studies have found higher rates of sensitivity for PET/CT in identifying the primary site (up to 67\%) as compared to historical rates reported from PET alone $[31,32]$, or with either PET or CT alone [33]. In the latter study, the imaging was interpreted by a team comprised of a nuclear medicine physician and a diagnostic radiologist [33]. Given the major changes in staging and therapy that result from identification of one or more head and neck primary sites, at our institution we recommend a dual-modality fused PET/CT including a diagnostic-quality head and neck contrast-enhanced CT, as part of the standard CUPS workup, ideally obtained prior to endoscopy and directed biopsies and/or bilateral tonsillectomy. We recommend evaluation by both a nuclear medicine and neuroradiology specialist to maximize the interpretative yield.

\section{Conclusions}

Skilled anatomic interpretation of PET/CT imaging led to the correct diagnosis of bilateral synchronous tonsillar squamous cell carcinoma. This case illustrates the expanding applications of PET/CT for head and neck cancer including the identification of occult or synchronous primaries in CUPS, detection of metastasis, and radiotherapy target identification. A properly executed PET/CT can greatly assist in the initial workup of patients with head and neck CUPS.

\section{Conflict of Interests}

The authors declare that there is no conflict of interests.

\section{References}

[1] S. J. McQuone, D. W. Eisele, D. J. Lee, W. H. Westra, and W. M. Koch, "Occult tonsillar carcinoma in the unknown primary," Laryngoscope, vol. 108, no. 11, pp. 1605-1610, 1998.

[2] W. M. Mendenhall, A. A. Mancuso, J. T. Parsons, S. P. Stringer, and N. J. Cassisi, "Diagnostic evaluation of squamous cell carcinoma metastatic to cervical lymph nodes from an unknown head and neck primary site," Head and Neck, vol. 20, no. 8, pp. 739-744, 1998.

[3] M. Lapeyre, L. Malissard, D. Peiffert et al., "Cervical lymph node metastasis from an unknown primary: is a tonsillectomy necessary?" International Journal of Radiation Oncology Biology Physics, vol. 39, no. 2, pp. 291-296, 1997.

[4] O. Guntinas-Lichius, J. P. Klussmann, S. Dinh et al., "Diagnostic work-up and outcome of cervical metastases from an unknown primary," Acta Oto-Laryngologica, vol. 126, no. 5, pp. 536-544, 2006.

[5] I. Kazak, A. Haisch, and S. Jovanovic, "Bilateral synchronous tonsillar carcinoma in cervical cancer of unknown primary site (CUPS)," European Archives of Oto-Rhino-Laryngology, vol. 260, no. 9, pp. 490-493, 2003.

[6] D. A. Randall, P. A. S. Johnstone, R. D. Foss, and P. J. Martin, "Tonsillectomy in diagnosis of the unknown primary tumor of the head and neck," Otolaryngology, vol. 122, no. 1, pp. 52-55, 2000.

[7] M. Cianchetti, A. A. Mancuso, R. J. Amdur et al., "Diagnostic evaluation of squamous cell carcinoma metastatic to cervical lymph nodes from an unknown head and neck primary site," Laryngoscope, vol. 119, no. 12, pp. 2348-2354, 2009.

[8] W. M. Koch, N. Bhatti, M. F. Williams, and D. W. Eisele, "Oncologic rationale for bilateral tonsillectomy in head and neck squamous cell carcinoma of unknown primary source," Otolaryngology, vol. 124, no. 3, pp. 331-333, 2001.

[9] J. D. Waltonen, E. Ozer, N. C. Hall, D. E. Schuller, and A. Agrawal, "Metastatic carcinoma of the neck of unknown primary origin: evolution and efficacy of the modern workup," Archives of Otolaryngology, vol. 135, no. 10, pp. 1024-1029, 2009.

[10] P. Kothari, P. S. Randhawa, and R. Farrell, "Role of tonsillectomy in the search for a squamous cell carcinoma from an unknown primary in the head and neck," British Journal of Oral and Maxillofacial Surgery, vol. 46, no. 4, pp. 283-287, 2008.

[11] J. L. Roh, J. S. Kim, J. H. Lee et al., "Utility of combined 18F-fluorodeoxyglucose-positron emission tomography and computed tomography in patients with cervical metastases from unknown primary tumors," Oral Oncology, vol. 45, no. 3, pp. 218-224, 2009.

[12] F. R. Miller, D. Hussey, M. Beeram, T. Eng, H. S. McGuff, and R. A. Otto, "Positron emission tomography in the management of unknown primary head and neck carcinoma," Archives of Otolaryngology, vol. 131, no. 7, pp. 626-629, 2005.

[13] N. Fakhry, T. Jacob, J. Paris et al., "Contribution of 18-FFDG PET for detection of head and neck carcinomas with an unknown primary tumor," Annales d'Oto-Laryngologie et de Chirurgie Cervico-Faciale, vol. 123, no. 1, pp. 17-25, 2006.

[14] T. Price and J. Pickles, "Synchronous bilateral tonsillar carcinoma: role of fluoro-deoxyglucose positron emission tomography scanning in detecting occult primary tumours in metastatic nodal disease of the head and neck," Journal of Laryngology and Otology, vol. 120, no. 4, pp. 334-337, 2006.

[15] K. Strobel, S. K. Haerle, S. J. Stoeckli et al., "Head and neck squamous cell carcinoma (HNSCC) — detection of synchronous primaries with 18F-FDG-PET/CT," European Journal of Nuclear Medicine and Molecular Imaging, vol. 36, no. 6, pp. 919-927, 2009.

[16] W. Shi, H. Kato, B. Perez-Ordonez et al., "Comparative prognostic value of HPV16 E6 mRNA compared with in situ hybridization for human oropharyngeal squamous carcinoma," Journal of Clinical Oncology, vol. 27, no. 36, pp. 62136221, 2009.

[17] K. K. Ang, J. Harris, R. Wheeler et al., "Human papillomavirus and survival of patients with oropharyngeal cancer," New England Journal of Medicine, vol. 363, no. 1, pp. 24-35, 2010.

[18] J. M. J. A. A. Straetmans, N. Olthof, J. J. Mooren, J. De Jong, E. J. M. Speel, and B. Kremer, "Human papillomavirus reduces the prognostic value of nodal involvement in tonsillar squamous cell carcinomas," Laryngoscope, vol. 119, no. 10, pp. 1951-1957, 2009.

[19] S. Begum, D. Cao, M. Gillison, M. Zahurak, and W. H. Westra, "Tissue distribution of human papillomavirus 16 DNA integration in patients with tonsillar carcinoma," Clinical Cancer Research, vol. 11, no. 16, pp. 5694-5699, 2005.

[20] S. L. McGovern, M. D. Williams, R. S. Weber et al., "Three synchronous HPV-associated squamous cell carcinomas of Waldeyer's ring: case report and comparison with Slaughter's model of field cancerization," Head and Neck, vol. 32, no. 8, pp. 1118-1124, 2010. 
[21] D. Chepeha and A. Eisbruch, "Commentary: clinical nodal staging of human papillomavirus-related oropharyngeal cancer," Cancer Journal, vol. 16, no. 3, p. 283, 2010.

[22] T. J. Kruser, K. A. Bradley, S. M. Bentzen et al., "The impact of hybrid PET-CT scan on overall oncologic management, with a focus on radiotherapy planning: a prospective, blinded study," Technology in Cancer Research and Treatment, vol. 8, no. 2, pp. 149-158, 2009.

[23] A. Guido, L. Fuccio, B. Rombi et al., "Combined 18F-FDGPET/CT imaging in radiotherapy target delineation for headand-neck cancer," International Journal of Radiation Oncology Biology Physics, vol. 73, no. 3, pp. 759-763, 2009.

[24] L. Deantonio, D. Beldì, G. Gambaro et al., "FDG-PET/CT imaging for staging and radiotherapy treatment planning of head and neck carcinoma," Radiation Oncology, vol. 3, no. 1, article 29, 2008.

[25] M. R. Vernon, M. Maheshwari, C. J. Schultz et al., "Clinical outcomes of patients receiving integrated PET/CT-guided radiotherapy for head and neck carcinoma," International Journal of Radiation Oncology Biology Physics, vol. 70, no. 3, pp. 678-684, 2008.

[26] S. A. Shintani, R. L. Foote, V. J. Lowe, P. D. Brown, Y. I. Garces, and J. L. Kasperbauer, "Utility of PET/CT imaging performed early after surgical resection in the adjuvant treatment planning for head and neck cancer," International Journal of Radiation Oncology Biology Physics, vol. 70, no. 2, pp. 322-329, 2008.

[27] G. D. R. Estrada-Sánchez, J. Altamirano-Ley, and F. J. OchoaCarrillo, "Normal variants and frequent pitfalls with (18)FDG PET/CT study," Cirugia y Cirujanos, vol. 75, no. 6, pp. 491497, 2007.

[28] F. U. Chowdhury, F. Sheerin, K. M. Bradley, and F. V. Gleeson, "Sarcoid-like reaction to malignancy on whole-body integrated 18F-FDG PET/CT: prevalence and disease pattern," Clinical Radiology, vol. 64, no. 7, pp. 675-681, 2009.

[29] S. K. Kim, H. J. Im, W. Kim, T. S. Kim, B. Hwangbo, and H. J. Kim, "F-18 fluorodeoxyglucose and F-18 fluorothymidine positron emission tomography/computed tomography imaging in a case of neurosarcoidosis," Clinical Nuclear Medicine, vol. 35, no. 2, pp. 67-70, 2010.

[30] K. Nassenstein, P. Veit-Haibach, H. Stergar et al., "Cervical lymph node metastases of unknown origin: primary tumor detection with whole-body positron emission tomography/computed tomography," Acta Radiologica, vol. 48, no. 10, pp. 1101-1108, 2007.

[31] C. Nanni, D. Rubello, P. Castellucci et al., "Role of 18F-FDG PET-CT imaging for the detection of an unknown primary tumour: preliminary results in 21 patients," European Journal of Nuclear Medicine and Molecular Imaging, vol. 32, no. 5, pp. 589-592, 2005.

[32] R. C. Delgado-Bolton, C. Fernández-Pérez, A. González-Maté, and J. L. Carreras, "Meta-analysis of the performance of 18FFDG PET in primary tumor detection in unknown primary tumors," Journal of Nuclear Medicine, vol. 44, no. 8, pp. 13011314, 2003.

[33] A. Gutzeit, G. Antoch, H. Kühl et al., "Unknown primary tumors: detection with dual-modality PET/CT-initial experience," Radiology, vol. 234, no. 1, pp. 227-234, 2005. 


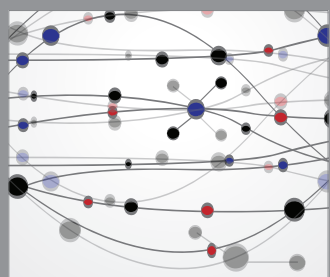

The Scientific World Journal
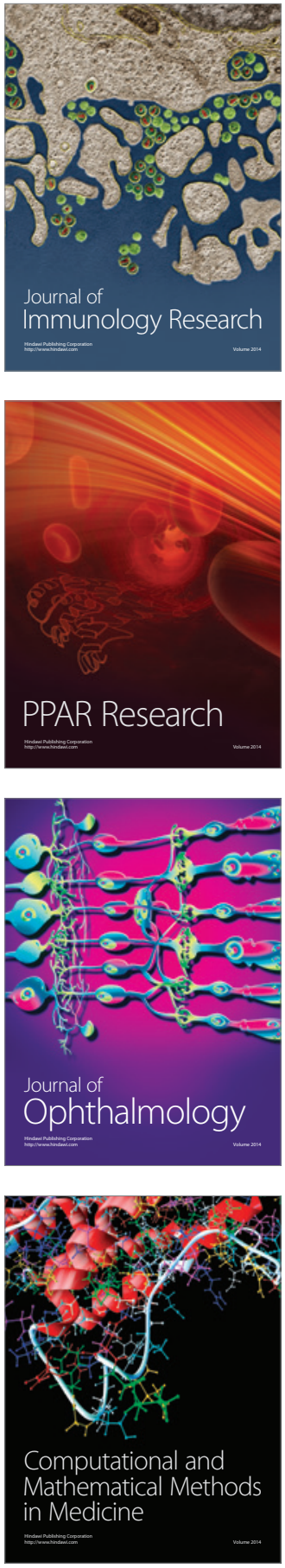

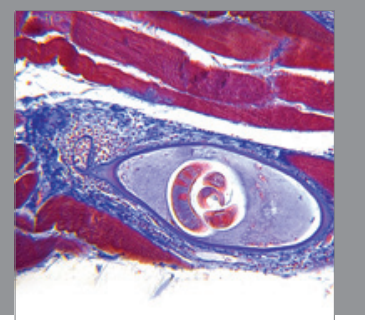

Gastroenterology

Research and Practice
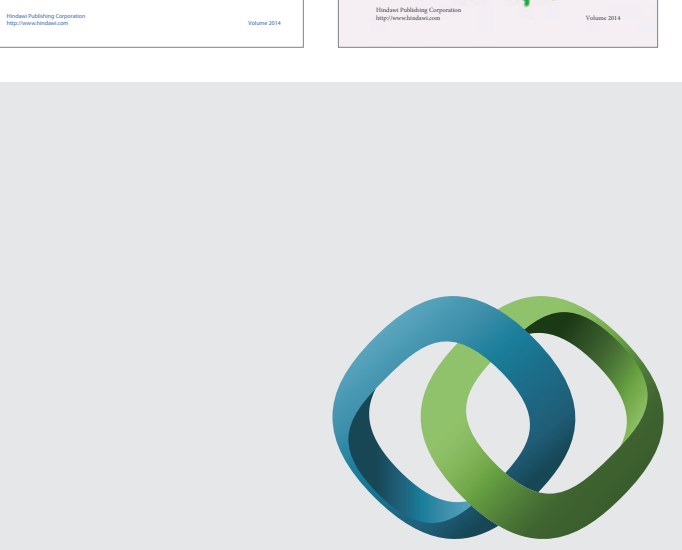

\section{Hindawi}

Submit your manuscripts at

http://www.hindawi.com
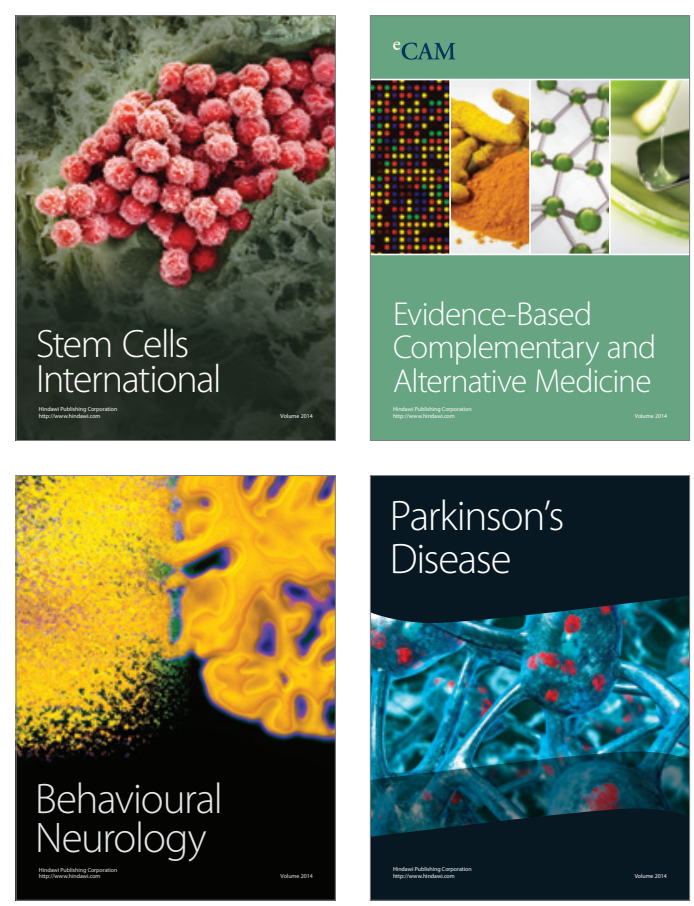

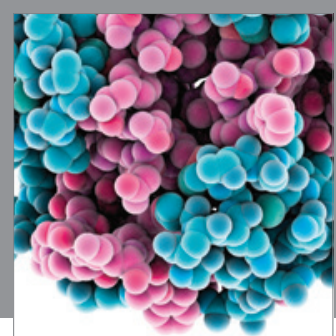

Journal of
Diabetes Research

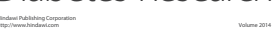

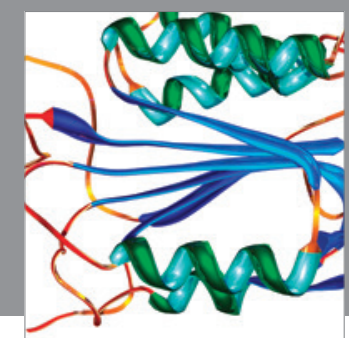

Disease Markers
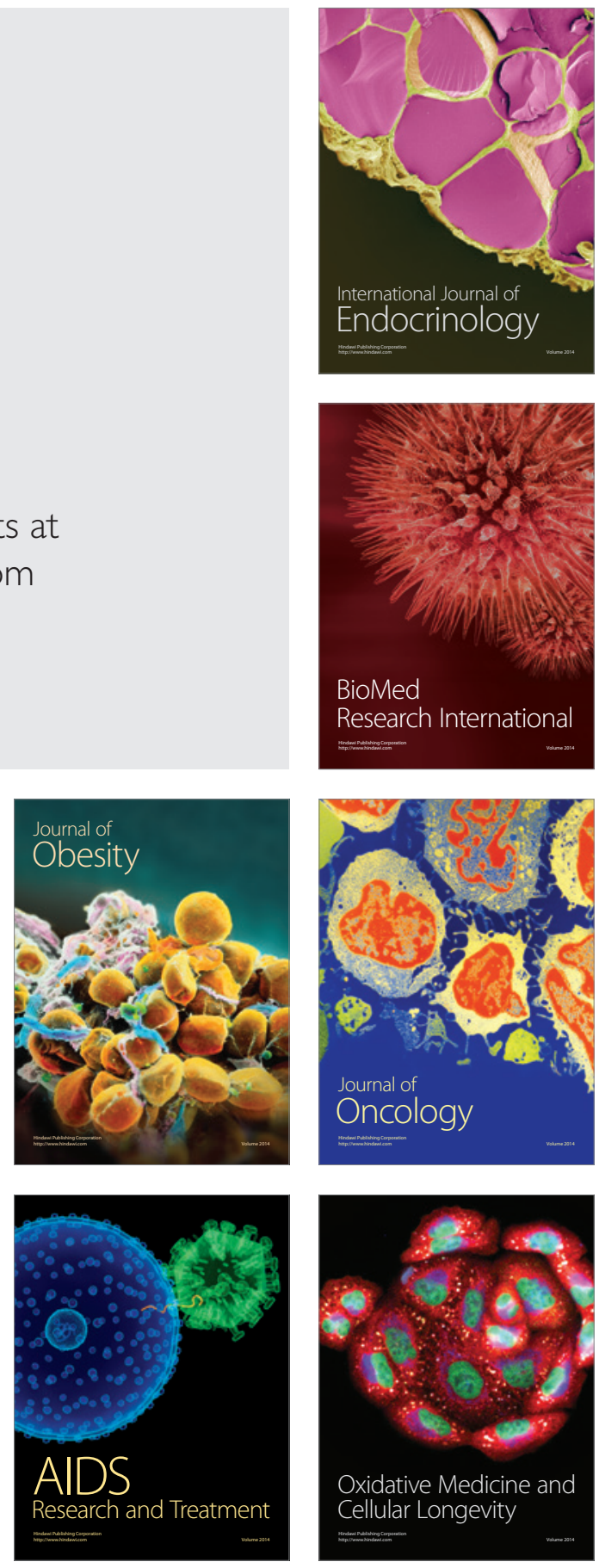